\title{
A Wideband Index Modulation with Circularly-Shifted Chirps
}

\author{
Safi Shams Muhtasimul Hoque*, Chao-Yu Chen ${ }^{\dagger}$, Alphan Şahin* \\ *Electrical Engineering Department, University of South Carolina, Columbia, SC, USA \\ ${ }^{\dagger}$ Department of Engineering Science, National Cheng Kung University, Tainan, Taiwan, R.O.C \\ E-mail: shoque@email.sc.edu, super@mail.ncku.edu.tw, asahin@mailbox.sc.edu
}

\begin{abstract}
In this study, we propose a wideband index modulation (IM) based on circularly-shifted chirps. To derive the proposed method, we first prove that a Golay complementary pair (GCP) can be constructed by linearly combining the Fourier series of chirps. We show that Fresnel integrals and/or Bessel functions, arising from sinusoidal and linear chirps, respectively, can lead to GCPs. We then exploit discrete Fourier transform-spread orthogonal frequency division multiplexing (DFT-s-OFDM) to obtain a low-complexity transmitter and receiver. We also discuss its generalization for achieving a trade-off between peak-to-mean envelope power ratio (PMEPR) and spectral efficiency (SE). Through comprehensive simulations, we compare the proposed scheme with DFT-s-OFDM with IM, orthogonal frequency division multiplexing (OFDM) with IM and complementary sequences (CSs) from Reed-Muller (RM) code. Our numerical results show that the proposed method limits the PMEPR while exploiting the frequency selectivity in fading channels without an auxiliary method.
\end{abstract}

Index Terms-Chirps, complementary sequences, index modulation, PMEPR

\section{INTRODUCTION}

Index modulation (IM) is a general modulation concept which disables or activates a set of resources in different dimensions (e.g., antennas, time slots, subcarriers, codes) while providing a flexible framework for traditional coherent modulation techniques. It has been considered for Fifth Generation $(5 \mathrm{G})$ networks as it can provide a trade-off between energy efficiency and spectral efficiency (SE) under an orthogonal frequency division multiplexing (OFDM) framework and spatial techniques [1], [2] (see also the references therein).

Within the last decade, OFDM with IM has been studied extensively, particularly for achieving a reliable high datarate transmission. For example, in [3], the subcarriers are partitioned into several subblocks and the specific indices of the active subcarriers in each subblock are used for data transmission. In [4], it is combined with an interleaver, where the real and imaginary parts of the complex data symbols are transmitted over different active subcarriers of the OFDMIM scheme. In [5], interleaved versus localized grouping is studied. In [6], repetition coding over multiple subcarriers and partitioned constellations are proposed to achieve a diversity gain. Nevertheless, it has been shown that IM is more beneficial for a low data-rate communication scenario as compared to typical OFDM transmission through a minimum Euclidean distance analysis [1]. Given this result, in this study, we target a reliable low data-rate transmission with IM where we can exploit the frequency selectivity naturally through wideband circularly-shifted chirp signals.

A chirp signal can sweep a large frequency spectrum over time. The efficacy of a chirp signal in a radar system was first demonstrated in [7] and later extended to the communications by encoding bits with negative and positive slopes of a linear chirp in the time-frequency (TF) plane. A low datarate communication through a proprietary chirp modulation, called Long Range (LoRa), was introduced by Samtec for Internet-of-Things (IoT) networks. To achieve a higher data rate, a chirp signal is translated in the frequency domain to achieve a set of orthogonal chirps [8]. In [9], circularly-shifted chirps are proposed by using the structure of discrete Fourier transform-spread orthogonal frequency division multiplexing (DFT-s-OFDM) with a specific frequency-domain spectral shaping (FDSS) function. Hence, a framework is established for chirps by using the typical OFDM blocks.

In this study, we consider circularly-shifted chirps with IM and exploit the framework in [9]. We provide both theoretical and practical contributions in various areas listed as follows:

- Chirps and complementary sequences (CSs): We prove that a CS [10] can be constructed by linearly combining the Fourier series of the chirp signals. Hence, two seemingly different topics are shown to be connected.

- Low-complexity framework: We show that the DFT-s-OFDM can be utilized to achieve a lowcomplexity wideband IM with chirps. We also generalize the proposed scheme to obtain a trade-off between peakto-mean envelope power ratio (PMEPR) and SE.

- Diversity gain: We show that the wideband nature of chirps exploits the frequency selectivity without any auxiliary method.

- Comprehensive comparisons: We compare the proposed scheme with OFDM-IM, DFT-s-OFDM-IM, and the CS from Reed-Muller (RM) codes [11], [12], numerically, in terms of PMEPR and error rate.

The rest of the paper is organized as follows. In Section II, preliminary discussions are provided. In Section III, the relationship between CSs and chirps are discussed. By using the results obtained in Section III, a low-complexity modulation scheme is derived. In Section $\nabla$ the proposed scheme is assessed numerically. The paper is concluded in Section VI.

Notation: The sets of complex numbers and positive integers are denoted by $\mathbb{C}$ and $\mathbb{Z}^{+}$, respectively. Conjugation is denoted 
by $(\cdot)^{*}$. The notation $\left(a_{i}\right)_{i=0}^{M-1}$ represents the sequence $\boldsymbol{a}=$ $\left(a_{0}, a_{1}, \ldots, a_{M-1}\right)$. The constant $\mathrm{j}$ denotes $\sqrt{-1}$.

\section{PREliminaries}

An OFDM symbol with the symbol duration $T_{\mathrm{s}}$ can be expressed in continuous time as a polynomial given by

$$
p_{\boldsymbol{a}}(z) \triangleq a_{M-1} z^{M-1}+a_{M-2} z^{M-2}+\cdots+a_{0},
$$

where $\boldsymbol{a}=\left(a_{0}, a_{1}, \ldots, a_{M}\right)$ is a sequence of length $M$, and $z \in\left\{\mathrm{e}^{\mathrm{j} \frac{2 \pi t}{T_{\mathrm{s}}}} \mid 0 \leq t<T_{\mathrm{s}}\right\}$.

\section{A. Circularly-shifted Chirps}

Let $B_{\tau_{m}}(t)=\mathrm{e}^{\mathrm{j} \psi_{m}(t)}$ be the $m$ th circular translation of an arbitrary band-limited function with the duration $T_{\mathrm{s}}$, where $\tau_{m}$ is the amount of circular shift and $m=0,1,2, \ldots, M-1$. By using Fourier series, $B_{\tau_{m}}(t)$ can be approximately expressed as

$$
B_{\tau_{m}}(t) \approx \sum_{k=L_{\mathrm{d}}}^{L_{\mathrm{u}}} c_{k} \mathrm{e}^{\mathrm{j} 2 \pi k \frac{t-\tau_{m}}{T_{\mathrm{s}}}}
$$

where $L_{\mathrm{d}}<0$ and $L_{\mathrm{u}}>0$ are integers, and $c_{k}$ is the $k$ th Fourier coefficient given by

$$
c_{k}=\mathcal{F}\left\{\mathrm{e}^{\mathrm{j} \psi_{0}(t)}\right\} \triangleq \frac{1}{T_{\mathrm{s}}} \int_{T_{\mathrm{s}}} \mathrm{e}^{\mathrm{j} \psi_{0}(t)} \mathrm{e}^{-\mathrm{j} 2 \pi k \frac{t}{T_{\mathrm{s}}}} d t .
$$

Let $D / 2 T_{\mathrm{s}}$ be the maximum frequency deviation around the carrier frequency. The approximation in (2) then becomes more accurate for $L_{\mathrm{d}}<-D / 2$ and $L_{\mathrm{u}}>D / 2$. This is due to the fact that $B_{\tau_{m}}(t)$ is a band-limited function, i.e., $\left|c_{k}\right|$ approaches zero rapidly for $|k|>D / 2$. Note that the actual bandwidth of a chirp is slightly larger than twice the maximum frequency deviation [13]. It can be calculated based on the total integrated power of the transmitted spectrum, i.e., occupied channel bandwidth (OCB). In this study, we express the OCB as $M_{\mathrm{ocb}} / T_{\mathrm{s}} \mathrm{Hz}$ where $M_{\mathrm{ocb}} \in \mathbb{Z}^{+}$. Also, the instantaneous frequency of $B_{\tau_{m}}(t)$ around the carrier frequency $f_{\mathrm{c}}$ can be obtained as $F_{m}(t)=\frac{1}{2 \pi} d \psi_{m}(t) / d t \mathrm{~Hz}$.

1) Linear Chirps: Let $F_{0}(t)$ be a linear function changing from $-\frac{D}{2 T_{\mathrm{s}}} \mathrm{Hz}$ to $\frac{D}{2 T_{\mathrm{s}}} \mathrm{Hz}$, i.e., $F_{0}(t)=\frac{D}{2 T_{\mathrm{s}}}\left(\frac{2 t}{T_{\mathrm{s}}}-1\right)$. The $k$ th Fourier coefficient can be calculated as

$$
c_{k}=\gamma_{k}\left(C\left(\alpha_{k}\right)+C\left(\beta_{k}\right)+\mathrm{j} S\left(\alpha_{k}\right)+\mathrm{j} S\left(\beta_{k}\right)\right),
$$

where $C(\cdot)$ and $S(\cdot)$ are the Fresnel integrals with cosine and sine functions, respectively, and $\alpha_{k}=(D / 2+2 \pi k) / \sqrt{\pi D}$, $\beta_{k}=(D / 2-2 \pi k) / \sqrt{\pi D}$, and $\gamma_{k}=\sqrt{\frac{\pi}{D}} \mathrm{e}^{-\mathrm{j} \frac{(2 \pi k)^{2}}{2 D}-\mathrm{j} \pi k}$ [9].

2) Sinusoidal Chirps: Let $F_{0}(t)=\frac{D}{2 T_{\mathrm{s}}} \cos \left(2 \pi \frac{t}{T_{\mathrm{s}}}\right)$. In this case, the maximum frequency deviation is $D / 2 T_{\mathrm{s}} \mathrm{Hz}$ and it can be shown that

$$
c_{k}=J_{k}\left(\frac{D}{2}\right)
$$

where $J_{k}(\cdot)$ is the Bessel function of the first kind of order $k$ [13].

\section{B. Chirps with DFT-s-OFDM}

Let complex baseband signal $p(t)$ be a linear combinations of the translated chirps as

$$
p(t)=\sum_{m=0}^{M-1} d_{m} B_{\tau_{m}}(t),
$$

where $d_{m} \in \mathbb{C}$ is the $m$ th modulation symbol, e.g., a phaseshift keying (PSK) symbol. In [9], it was shown that if $\tau_{m}=$ $m / M \times T_{\mathrm{s}}$ (i.e., uniform spacing in time), $p(t)$ in discrete time can be written as

$$
p\left(\frac{n T_{\mathrm{s}}}{N}\right) \approx \sum_{k=L_{\mathrm{d}}}^{L_{\mathrm{u}}} c_{k} \sum_{m=0}^{M-1} d_{m} \mathrm{e}^{-\mathrm{j} 2 \pi k \frac{m}{M}} \mathrm{e}^{\mathrm{j} 2 \pi k \frac{n}{N}},
$$

by sampling $p(t)$ with the period $T_{\mathrm{s}} / N$. Equation (7) shows that chirps signal can be synthesized by using the DFT-s-OFDM transmitter with a special choice of FDSS sequence which substantially reduces the transmitter complexity. Since a typical DFT-s-OFDM receiver with a singletap minimum mean square error (MMSE) frequency-domain equalization (FDE) can be utilized [14], it also offers a low-complexity at the receiver side. Note that the condition $M \geq M_{\text {ocb }}>D$ must hold to be able to represent a chirp by using $M$ subcarriers. In this study, we also consider that $L_{\mathrm{u}}-L_{\mathrm{d}}+1=M$ to avoid FDSS beyond $M$ subcarriers.

\section{Complementary Sequences}

The sequence pair $(\boldsymbol{a}, \boldsymbol{b})$ of length $M$ is a Golay complementary pair (GCP) if $\rho_{\boldsymbol{a}}(k)+\rho_{\boldsymbol{b}}(k)=0$ for $k \neq 0$ where $\rho_{\boldsymbol{a}}(k)$ and $\rho_{\boldsymbol{b}}(k)$ are the aperiodic autocorrelations (APACs) of the sequences $\boldsymbol{a}$ and $\boldsymbol{b}$ at the $k$ th lag, respectively [10]. Each sequence in a GCP is called a CS. A GCP $(\boldsymbol{a}, \boldsymbol{b})$ can also be defined as any sequence pair satisfying $\left|p_{\boldsymbol{a}}\left(\mathrm{e}^{\mathrm{j} \frac{2 \pi t}{T_{\mathrm{s}}}}\right)\right|^{2}+\left|p_{\boldsymbol{b}}\left(\mathrm{e}^{\mathrm{j} \frac{2 \pi t}{T_{\mathrm{s}}}}\right)\right|^{2}=\rho_{\boldsymbol{a}}(0)+\rho_{\boldsymbol{b}}(0)[15]$.

If a CS is transmitted with an OFDM waveform, the instantaneous peak power of the corresponding signal is bounded, i.e., $\max _{t}\left|p_{\boldsymbol{a}}\left(\mathrm{e}^{\mathrm{j} \frac{2 \pi t}{T_{\mathrm{s}}}}\right)\right|^{2} \leq \rho_{\boldsymbol{a}}(0)+\rho_{\boldsymbol{b}}(0)$. As a result, the PMEPR of the OFDM symbol $p_{\boldsymbol{a}}\left(\mathrm{e}^{\mathrm{j} \frac{2 \pi t}{T_{\mathrm{s}}}}\right)$, defined as $\max _{t}\left|p_{\boldsymbol{a}}\left(\mathrm{e}^{\mathrm{j} \frac{2 \pi t}{T_{\mathrm{s}}}}\right)\right|^{2} / P_{\mathrm{av}}$, is less than or equal to $10 \log _{10}(2) \approx$ $3 \mathrm{~dB}$ if $P_{\mathrm{av}}=\rho_{\boldsymbol{a}}(0)=\rho_{\boldsymbol{b}}(0)$. Note that for non-unimodular CSs, $\rho_{\boldsymbol{a}}(0)$ may not be equal to $\rho_{\boldsymbol{b}}(0)$. In that case, the power of OFDM symbol with $\boldsymbol{a}$ can be different from the one with $\boldsymbol{b}$ although the instantaneous peak power is still less than or equal to $\rho_{\boldsymbol{a}}(0)+\rho_{\boldsymbol{b}}(0)$ for both symbols. Hence, to avoid misleading results, we define $P_{\mathrm{av}}$ as the power of the complex baseband signal in this study.

\section{Chirp-Based Complementary SEQUenCeS}

Theorem 1. Let $x(t)$ and $y(t)$ be two complex-valued signals defined by

$$
\begin{aligned}
& x(t)=d_{m} \mathrm{e}^{\mathrm{j} \psi_{m}(t)}+d_{n} \mathrm{e}^{\mathrm{j} \psi_{n}(t)}, \\
& y(t)=d_{m} \mathrm{e}^{\mathrm{j} \psi_{m}(t)}-d_{n} \mathrm{e}^{\mathrm{j} \psi_{n}(t)},
\end{aligned}
$$

where $d_{m}, d_{n} \in \mathbb{C}$ and $\left|d_{m}\right|=\left|d_{n}\right|=1$. The Fourier coefficients of $x(t)$ and $y(t)$ form a GCP. 
Proof. By the definition, we need to show that $|x(t)|^{2}+|y(t)|^{2}$ is constant:

$$
\begin{aligned}
|x(t)|^{2}= & \left|d_{m}\right|^{2}+\left|d_{n}\right|^{2} \\
& +d_{m} d_{n}^{*} \mathrm{e}^{\mathrm{j}\left(\psi_{m}(t)-\psi_{n}(t)\right)}+d_{m}^{*} d_{n} \mathrm{e}^{-\mathrm{j}\left(\psi_{m}(t)-\psi_{n}(t)\right)} .
\end{aligned}
$$

Similarly,

$$
\begin{aligned}
|y(t)|^{2}= & \left|d_{m}\right|^{2}+\left|d_{n}\right|^{2} \\
& -d_{m} d_{n}^{*} \mathrm{e}^{\mathrm{j}\left(\psi_{m}(t)-\psi_{n}(t)\right)}-d_{m}^{*} d_{n} \mathrm{e}^{-\mathrm{j}\left(\psi_{m}(t)-\psi_{n}(t)\right)} .
\end{aligned}
$$

Therefore, $|x(t)|^{2}+|y(t)|^{2}=2 \times\left(\left|d_{m}\right|^{2}+\left|d_{n}\right|^{2}\right)=4$, which implies that $\mathcal{F}\{x(t)\}$ and $\mathcal{F}\{y(t)\}$ form a GCP.

Theorem 1 indicates that the Fourier coefficients of a linear combination of the frequency response of two constantenvelope chirps result in a CS. As a result, it yields an interesting connection between chirps and CSs.

Example 1. Assume that $x(t)$ and $y(t)$ are linear combinations of two circularly-shifted versions of a band-limited sinusoidal chirp defined in Section III-A2 Therefore, by using (2) and (5), the Fourier coefficients of $x(t)$ and $y(t)$ are obtained as

$$
\begin{aligned}
& a_{k}=d_{m} J_{k}\left(\frac{D}{2}\right) \mathrm{e}^{-\mathrm{j} 2 \pi k \frac{\tau_{m}}{T_{\mathrm{s}}}}+d_{n} J_{k}\left(\frac{D}{2}\right) \mathrm{e}^{-\mathrm{j} 2 \pi k \frac{\tau_{n}}{T_{\mathrm{s}}}}, \\
& b_{k}=d_{m} J_{k}\left(\frac{D}{2}\right) \mathrm{e}^{-\mathrm{j} 2 \pi k \frac{\tau_{m}}{T_{\mathrm{s}}}}-d_{n} J_{k}\left(\frac{D}{2}\right) \mathrm{e}^{-\mathrm{j} 2 \pi k \frac{\tau_{n}}{T_{\mathrm{s}}}},
\end{aligned}
$$

respectively. Based on Theorem 1] $\left(a_{i}\right)_{i=-\infty}^{\infty}$ and $\left(b_{i}\right)_{i=-\infty}^{\infty}$ form a GCP. Since the sinusoidal chirps are band-limited signals, the amplitude of a Fourier coefficient approaches to zero for $|i| \geq D / 2$. Therefore, $\left(a_{i}\right)_{i=L_{\mathrm{d}}}^{L_{\mathrm{u}}}$ and $\left(b_{i}\right)_{i=L_{\mathrm{d}}}^{L_{\mathrm{u}}}$ are approximately GCP. Note that if the sinusoidal chirps are replaced by the linear chirps, by using (4), the Fourier coefficients of $x(t)$ and $y(t)$ can be calculated as

$$
\begin{aligned}
a_{k}= & d_{m} \gamma_{k}\left(C\left(\alpha_{k}\right)+C\left(\beta_{k}\right)+\mathrm{j} S\left(\alpha_{k}\right)+\mathrm{j} S\left(\beta_{k}\right)\right) \mathrm{e}^{-\mathrm{j} 2 \pi k \frac{\tau_{m}}{T_{\mathrm{s}}}} \\
& +d_{n} \gamma_{k}\left(C\left(\alpha_{k}\right)+C\left(\beta_{k}\right)+\mathrm{j} S\left(\alpha_{k}\right)+\mathrm{j} S\left(\beta_{k}\right)\right) \mathrm{e}^{-\mathrm{j} 2 \pi k \frac{\tau_{n}}{T_{\mathrm{s}}}}, \\
b_{k}= & d_{m} \gamma_{k}\left(C\left(\alpha_{k}\right)+C\left(\beta_{k}\right)+\mathrm{j} S\left(\alpha_{k}\right)+\mathrm{j} S\left(\beta_{k}\right)\right) \mathrm{e}^{-\mathrm{j} 2 \pi k \frac{\tau_{m}}{T_{\mathrm{s}}}} \\
& -d_{n} \gamma_{k}\left(C\left(\alpha_{k}\right)+C\left(\beta_{k}\right)+\mathrm{j} S\left(\alpha_{k}\right)+\mathrm{j} S\left(\beta_{k}\right)\right) \mathrm{e}^{-\mathrm{j} 2 \pi k \frac{\tau_{n}}{T_{\mathrm{s}}}} .
\end{aligned}
$$

In Figure 11 we exemplify a GCP of length $M=24$, synthesized through (10) and (11) for $L_{\mathrm{d}}=-11, L_{\mathrm{u}}=12$, $\tau_{m} / T_{\mathrm{s}}=0 / 24, \tau_{n} / T_{\mathrm{s}}=1 / 24$, and $d_{m}=d_{n}=1$. When $D=24$, the sequences are truncated heavily. Therefore, it does not satisfy the definition of a GCP given in Section $\amalg-C$ On the other hand, when the maximum frequency deviation is halved, $M_{\mathrm{ocb}}$ is 15 for containing $99 \%$ of the total integrated power of the spectrum. Hence, $M=24$ forms the chirps well and the resulting sequences form a GCP. It is also worth noting that synthesized CSs are not unimodular sequences. Therefore, the mean power of OFDM symbol changes although instantaneous power is bounded.

Corollary 2. Let the coefficients $d_{m}, d_{n} \in \mathbb{S}_{\mathrm{PSK}, H} \triangleq$ $\left\{\mathrm{e}^{\mathrm{j} 2 \pi \times \frac{k}{H}} \mid k=0,1, \ldots, H-1\right\}$. Let $\mathbb{S}_{\text {chirp }} \triangleq\left\{B_{\tau_{m}}(t) \mid m=\right.$ $0,1, \ldots, M-1\}$ be a set of $M$ uniformly circularly-shifted chirps of an arbitrary band-limited function with the duration
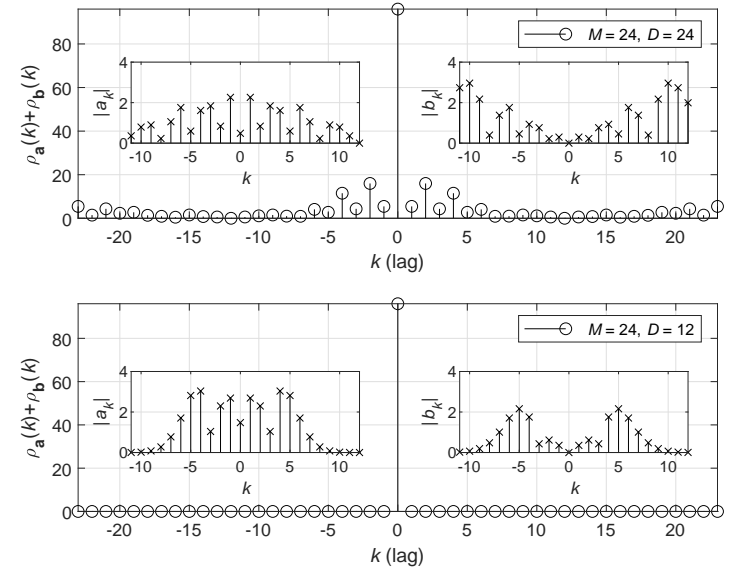

Figure 1. An instance of GCP of length $M=24$ synthesized through sinusoidal chirps.

$T_{\mathrm{S}}$ and $M_{\mathrm{ocb}} \leq M$. Without using the same chirp twice, the total number of distinct CSs of length $M$ is $\left(\begin{array}{c}M \\ 2\end{array}\right) \times H^{2}$.

Proof. Since $\left|\mathbb{S}_{\mathrm{PSK}, H}\right|=H$, there exist $H^{2}$ combinations for $\left\{d_{m}, d_{n}\right\}$. As $\left|\mathbb{S}_{\text {chirp }}\right|=M, \mathrm{e}^{\mathrm{j} \psi_{m}(t)}$ and $\mathrm{e}^{\mathrm{j} \psi_{n}(t)}$ in $(8)$ can be chosen in $\left(\begin{array}{c}M \\ 2\end{array}\right)$ ways without using the same chirp. Thus, the total number of CSs is $\left(\begin{array}{c}M \\ 2\end{array}\right) \times H^{2}$ via Theorem 11 The CSs are distinct as $B_{\tau_{m}}(t)$ are distinct. Since the OCB of $B_{\tau_{m}}(t)$ is less than or equal to $M / T_{\mathrm{s}}$, the length of the synthesized CS is $M$ based on Nyquist's sampling theorem.

\section{A Low-Complexity SCHEME WITH DFT-S-OFDM}

In this section, we utilize Corollary 2 to develop a wideband IM by using the structure of DFT-s-OFDM discussed in Section [II-B At the transmitter, we consider $S=$ $\left\lfloor\log _{2}\left(\left(\begin{array}{c}M \\ 2\end{array}\right) \times H^{2}\right)\right\rfloor$ information bits. Assuming $H$ is an integer power-of-two, we map $2 \log _{2}(H)$ of the information bits to $s_{1}$ and $s_{2}$, where $s_{1}, s_{2} \in \mathbb{S}_{\mathrm{PSK}, H}$. The rest of the information bits are utilized to choose a set $\{m, n\}$, where $m, n \in$ $\{0,1, \ldots, M-1\}$ and $m \neq n$. We generate the modulation symbols as $d_{m}=s_{1}, d_{n}=s_{2}$, and $d_{i \mid i \in\{0,1, \ldots, M-1\}, i \neq m, n}=$ 0 . We then synthesize the transmitted signal according to (7).

In Figure 2, we give the transmitter and receiver block diagrams. First, $M$-point discrete Fourier transform (DFT) of the modulation symbols $\left(d_{0}, d_{1}, \ldots, d_{M-1}\right)$ is calculated. The resulting sequence is then shaped in the frequency domain with an FDSS for generating chirps. According to Corollary 2 , after FDSS, a CS is formed since there are only two modulation symbols for the proposed scheme. We then map the resulting CS to the OFDM subcarriers. After an $N$-point inverse DFT (IDFT) of the mapped CS, the resulting signal is transmitted with a cyclic prefix $(\mathrm{CP})$.

At the receiver side, we consider a single-tap MMSE-FDE and a maximum-likelihood (ML) detector for detecting $m, n$, $s_{1}$, and $s_{2}$. Let $\left(\tilde{d}_{0}, \tilde{d}_{1}, \ldots, \tilde{d}_{M-1}\right)$ be the received modulation symbols after $M$-point IDFT operation. Since $\left|s_{1}\right|=\left|s_{2}\right|=1$, 


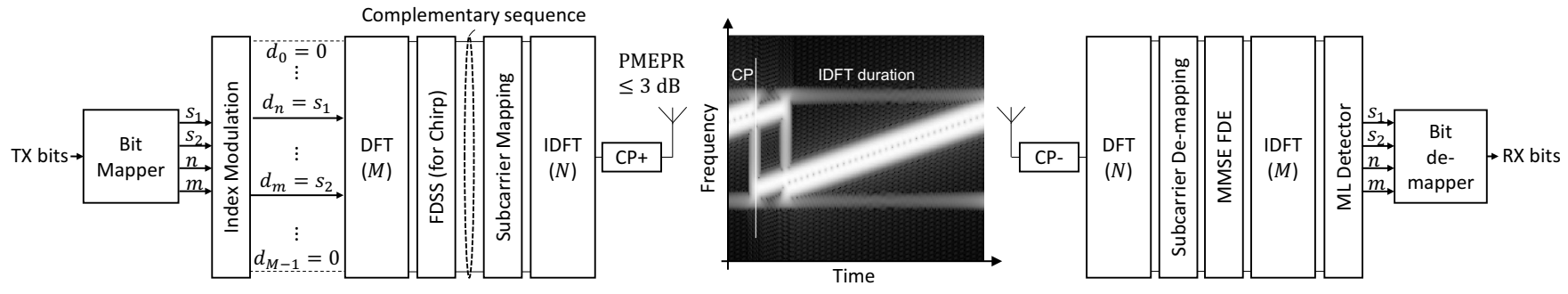

Figure 2. Transmitter and receiver block diagrams for the proposed scheme.

the ML detector for $m, n, s_{1}$, and $s_{2}$ can be expressed as

$$
\left\{\{\hat{m}, \hat{n}\}, \hat{s}_{1}, \hat{s}_{2}\right\}=\arg \max _{\substack{\{m, n\}, s_{1}, s_{2} \\ m \neq n}} \Re\left\{\tilde{d}_{m} s_{1}^{*}+\tilde{d}_{n} s_{2}^{*}\right\} .
$$

As $m \neq n$, a low-complexity ML detector can be implemented by evaluating $C_{i, k}=\Re\left\{\tilde{d}_{i} \mathrm{e}^{-\mathrm{j} 2 \pi k / H}\right\}$ for $i=0,1, \ldots, M-1$ and $k=0,1, \ldots, H-1$ and choosing two different indices for $i$ and the corresponding $k$ 's that maximize $C_{i, k}$.

\section{A. Generalizations and Practical Issues}

For a practical radio, the mapping from the information bits to a combination of $\{m, n\}$ at the transmitter (and vice versa for the receiver) may be a challenge. This can be addressed by constructing a bijection function from integers to the set of combinations via a combinatorial number system of degree 2 [3], also called combinadics.

The choice of FDSS is important for obtaining a good bit-error ratio (BER) performance and a low PMEPR. For example, a linear chirp offers a more flat FDSS as compared to the one with a sinusoidal chirp. In [9], it was demonstrated that a flatter FDSS improves the BER performance for the receiver with a single-tap MMSE-FDE. On the other hand, a linear chirp causes abrupt instantaneous frequency changes within the IDFT duration. Therefore, it requires a much lower $D$ as compared to the one for a sinusoidal chirp for a given $M$. This issue can distort the targeted CSs and cause a larger PMEPR than the theoretical bound, as demonstrated in Section $\nabla$

If more than two indices are allowed to be utilized, the proposed scheme can be generalized to a scheme that offers a trade-off between maximum PMEPR and SE. Let $l$ denote the number of indices that are allowed to be used. The SE of the scheme can be calculated as $\rho=$ $\frac{\left\lfloor\log _{2}\left(\left(\begin{array}{c}M \\ l\end{array}\right) \times H^{l}\right)\right\rfloor}{M} \mathrm{bit} / \mathrm{second} / \mathrm{Hz}$, while the PMEPR of a signal is always less than or equal to $l$ as $l$ chirps are transmitted simultaneously. In Figure 3, we illustrate the trade-off between the maximum PMEPR and the SE for a given $M$ and $H=4$ by changing $l$ from 1 to 11 . The generalized scheme reduces to the scheme in Figure 2 with CSs for $l=2$. If there is room for more transmit power, $l>2$ can be utilized for increasing the SE while still limiting PMEPR.

\section{B. Comparisons}

As compared to DFT-s-OFDM-IM, the proposed scheme has a PMEPR advantage since the signal is spread in the time domain whereas DFT-s-OFDM generates sinc pulses.

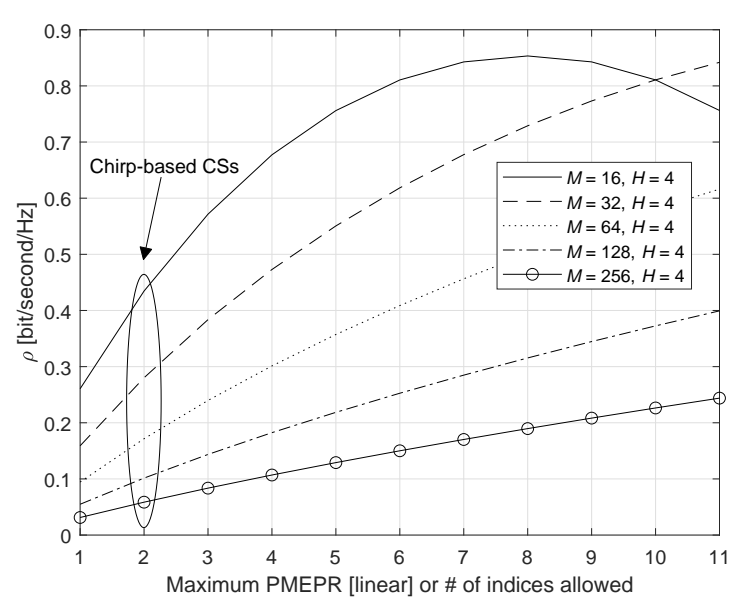

Figure 3. Trade-off between SE and maximum PMEPR.

The proposed scheme and OFDM with IM have similar PMEPR characteristics since OFDM with IM also spreads the symbol energy in time. However, the energy is also distributed within the signal bandwidth with the proposed scheme. Thus, the proposed scheme allows a coherent receiver to exploit frequency diversity in selective channels naturally. On the other hand, OFDM-IM receiver does not fully benefit from the frequency selectivity without an extra operation, e.g., repetitions or interleaving, at the transmitter.

In [11], a low PMEPR coding scheme was proposed to generate CSs from RM code. This scheme synthesizes $H^{m+1} \cdot m ! / 2$ CSs where the length of each CS has to be in the form of $2^{m}$, where $m \in \mathbb{Z}^{+}$. When a seed GCP of length $N$ is utilized with this scheme, it can be shown that $H^{m+1} \cdot m$ ! CSs of length $N \cdot 2^{m}$ can be generated [12]. Therefore, the spectral efficiency of the schemes in [11] and [12] can be calculated as $\left\lfloor\log _{2}\left(H^{m+1} \cdot m !\right)\right\rfloor / 2^{m+1}$ and $\left\lfloor\log _{2}\left(H^{m+1} \cdot m !\right)\right\rfloor /\left(N 2^{m}\right)$ $\mathrm{bit} / \mathrm{second} / \mathrm{Hz}$, respectively.

The differences between these schemes and the proposed scheme can be listed as follows: 1) The proposed scheme allows the length of CS to be chosen arbitrarily. For example, $M$ can be an integer chosen as an integer multiple of 12 based on the resource allocation in 3GPP 5G New Radio (NR) and Fourth Generation (4G) LTE or the resource units in IEEE 802.11ax, e.g., 26 subcarriers. 2) The schemes in [11], [12] do not offer a trade-off between PMEPR and spectral efficiency whereas $l$ can be chosen for a higher SE at a 
cost of high PMEPR with our scheme. The PMEPR is still theoretically limited. 3) The number of CSs is a function of a second order coset term generated through permutations in [11] and [12]. However, designing a decoder for all possible permutations is not trivial [16]. For a fixed coset, the decoder can be implemented through fast Hadamard transformation or recursive methods [17], but the SE reduces to $\left\lfloor\log _{2} H^{m+1}\right\rfloor$. Under this case, the SE of the proposed scheme and the schemes in [11] and [12] are similar while a simple decoder can be employed for the proposed method.

Note that the SE of the proposed scheme can be considered as low as compared to typical coding schemes such as lowdensity parity check (LDPC) or polar codes. Although this appears as a disadvantage, there exist communication scenarios (e.g., uplink control channels in 5G NR [12], IoT networks, and dual-function radar and communication (DFRC) scenarios) where the primary concern is reliability under low signalto-noise ratio (SNR) or a longer battery life, rather than a high data rate. For these scenarios, the proposed scheme provides a way of limiting PMEPR without an optimization procedure at the transmitter while exploiting frequency selectivity.

\section{Numerical Results}

For computer simulations, the symbol duration and the $\mathrm{CP}$ duration are set to $T_{\mathrm{s}}=16.67 \mu \mathrm{s}$ and $T_{\mathrm{cp}}=2.34 \mu \mathrm{s}$, respectively, based on the $5 \mathrm{G} \mathrm{NR}$ waveform parameters. We assume that the transmitter uses 32 resource blocks, i.e., $M=384$ subcarriers are used. The data symbols were generated based on quadrature phase-shift keying (QPSK), i.e., $H=4$. For the fading channel, we consider ITU Extended Vehicular A (EVA). At the transmitter, the combinations $\{m, n\}$ are generated by mapping natural order of integers to combinations and we choose $D=300$. At the receiver, all possible $\{m, n\}$ combinations are considered and a non-valid combination (e.g., due to the noise) is mapped to a valid combination to avoid catastrophic results. The FDSS is chosen based on (5) for sinusoidal chirps and (4) for linear chirps. We assume that the channel and FDSS information are available at the receiver. We compare the proposed scheme with OFDM-IM, DFT-s-OFDM-IM (i.e., no FDSS is applied), and the CSs from RM codes [11], [12]. For OFDM-IM, an ML detector which incorporates the channel frequency response is utilized [3]. For CSs from RM codes, we use a seed GCP of length $N=3$ and use $m=7$. To achieve the same spectral efficiency, we consider 16 permutations for 4 extra bits transmission and the ML decoder proposed in [17] (channel frequency response is also incorporated) is employed for each permutation.

In Figure 4, we plot the time-domain signals obtained with DFT-s-OFDM-IM and the proposed scheme for $l=2$. The DFT-s-OFDM-IM shows very high PMEPR as compared to the one generated with the proposed scheme as the indices are represented as Dirichlet-sinc pulses. With sinusoidal chirps, the instantaneous signal power never exceeds 2 (i.e., $\sqrt{2}$ as amplitude). This can be understood either by the properties of a CS or the summation of two circularly-shifted chirps. Similar behavior is also observed for linear chirps.

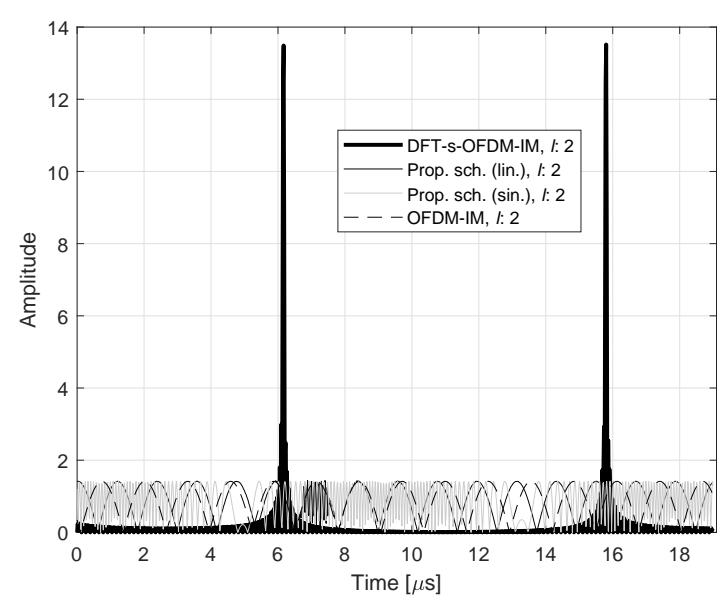

Figure 4. An instance of temporal behavior of the signals.

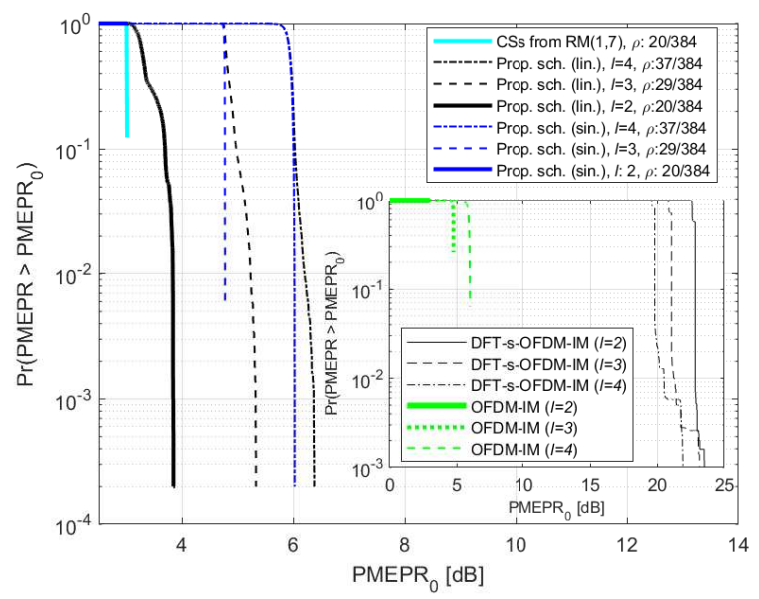

Figure 5. PMEPR distribution.

In Figure 5, the PMEPR distributions are provided for different schemes. The signal is over-sampled to measure PMEPR accurately. The PMEPR is always less than or equal to $3 \mathrm{~dB}$ for CSs from RM codes and sinusoidal chirps for $l=2$. However, the distortion on linear chirps due to the truncation is higher than the one for sinusoidal chirps for $D=300$. Therefore, the CS is not accurately formed with linear chirps under our simulation settings and the maximum PMEPR is slightly higher than $3 \mathrm{~dB}$. For $l=3$ and $l=4$, PMEPRs are still limited for the proposed scheme while offering a higher SE as compared to CSs from RM codes. OFDM-IM results in PMEPR distributions are similar to the ones for the proposed scheme. On the other hand, the DFT-s-OFDM-IM causes signals with very high PMEPRs.

In Figure 6 and Figure 7, we compare the error-rate performance in AWGN and fading channels for $l=\{2,4\}$. Since the proposed scheme utilizes IM and the DFT is an orthogonal transformation, it inherits the structural properties of orthogonal frequency-shift keying (FSK) with coherent detection at the receiver [13]. For the proposed scheme, the receiver equalizes the signal in the frequency domain even in the AWGN channel because of FDSS. Therefore, a flatter 


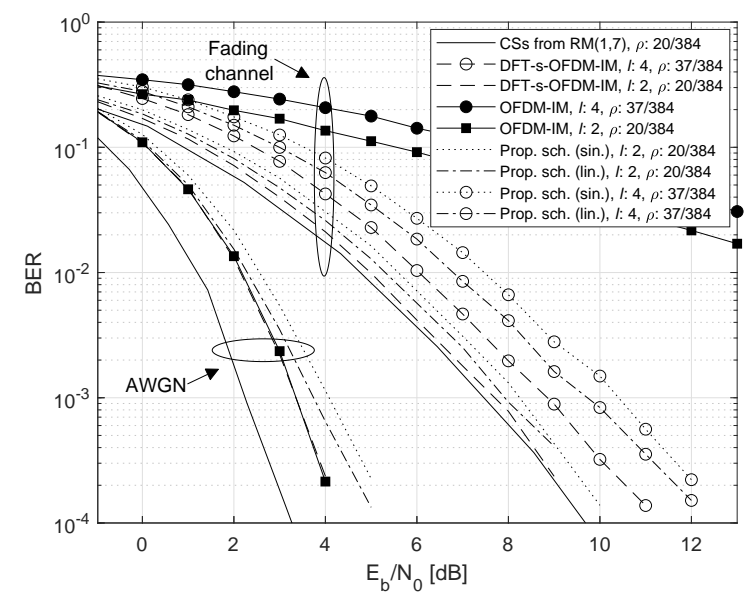

Figure 6. BER versus $E_{\mathrm{b}} / N_{0}$ in AWGN and fading channels.

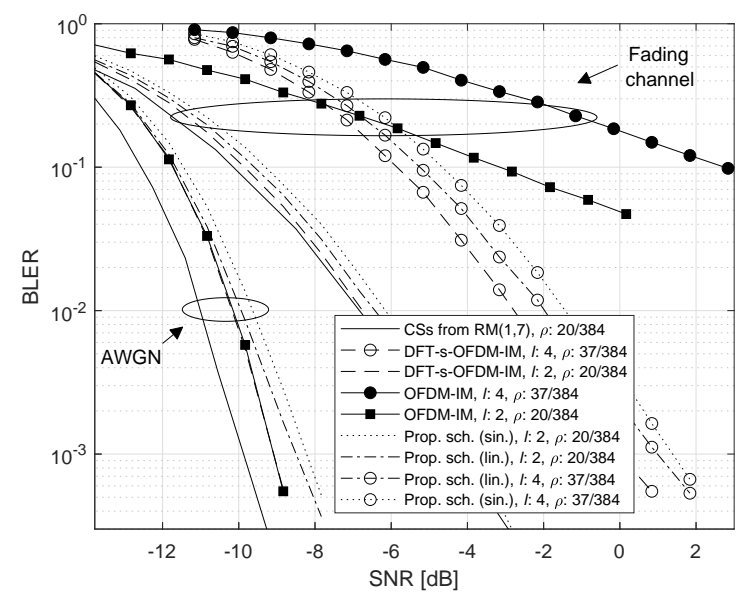

Figure 7. BLER versus SNR in AWGN and fading channels.

response improves the BER result in both AWGN and fading channels as demonstrated in Figure 6, Since FDSS for linear chirp is flatter than that of sinusoidal chirp [9], the proposed scheme with linear chirps yields better a BER performance. In AWGN, the proposed schemes with different FDSS operate in the range of $3.5 \mathrm{~dB}-4.5 \mathrm{~dB}$ for $E_{\mathrm{b}} / N_{0}$ at $1 \mathrm{e}-3$ BER. The CSs from RM code is superior to all other schemes for $l=2$ and provides $1.5 \mathrm{~dB}$ gain. However, this gain diminishes in the fading channel and the schemes result in similar performances. The maximum difference is around $0.5 \mathrm{~dB}$ at $E_{\mathrm{b}} / N_{0}=8 \mathrm{~dB}$ approximately for $l=2$. Note that the ML detector for CSs from RM code runs the recursive algorithm for 16 times for different permutations while the proposed scheme only relies on a single $M$-fast Fourier transform (FFT). For $l=4$, the proposed scheme offers flexibility to transmit a larger number of bits at the cost of $3 \mathrm{~dB}$ PMEPR increment. Increasing the number of transmitted bits from 20 to 37 causes approximately 4 dB SNR loss as shown in Figure 7 as the received energy per bit is approximately halved. Although the DFT-s-OFDM-IM performs $1 \mathrm{~dB}$ better than the proposed schemes for $l=4$, a larger power back-off is required for plain DFT-s-OFDM (see PMEPR distributions in Figure 5), which substantially offset this gain. The performance of OFDM-IM is worse than the proposed scheme since it does not fully exploit the frequency selectivity. The slopes of the BER and BLER curves for the proposed scheme under the fading channel are also noticeably higher than the ones for OFDM-IM as the transmitted signals are wideband.

\section{CONCLUding REMARKS}

In this study, we prove that CSs and chirps are related to each other. We exemplify that Bessel functions and Fresnel integrals can be useful for generating GCPs. By utilizing this relationship, we then develop a low-complexity low-PMEPR wide-band IM with chirps. We discuss a generalization of the proposed scheme, which yields a trade-off between SE and PMEPR. We compare the proposed scheme with OFDM-IM, DFT-s-OFDM-IM, and the CSs from RM codes. The proposed scheme offers significantly better PMEPR performance than DFT-s-OFDM-IM while exploiting frequency selectivity without any other additional method. Since it does not utilize a coset term needed for the CS from RM, a low-complexity decoder can also be utilized at the receiver for the proposed scheme.

\section{REFERENCES}

[1] N. Ishikawa, S. Sugiura, and L. Hanzo, "Subcarrier-index modulation aided OFDM - will it work?" IEEE Access, vol. 4, pp. 2580-2593, May 2016.

[2] X. Cheng, M. Zhang, M. Wen, and L. Yang, "Index modulation for 5G: Striving to do more with less," IEEE Wireless Communications, vol. 25, no. 2, pp. 126-132, May 2018.

[3] E. Başar, Ü. Aygölü, E. Panayırcı, and H. V. Poor, "Orthogonal frequency division multiplexing with index modulation," IEEE Trans. Signal Process., vol. 61, no. 22, pp. 5536-5549, Apr. 2013.

[4] E. Başar, "OFDM with index modulation using coordinate interleaving," IEEE Wireless Commun. Lett., vol. 4, no. 4, pp. 381-384, Aug. 2015.

[5] M. Wen, X. Cheng, M. Ma, B. Jiao, and H. V. Poor, "On the achievable rate of OFDM with index modulation," IEEE Trans. Signal Process. vol. 64, no. 8, pp. 1919-1932, Nov. 2016.

[6] A. T. Doğukan and E. Başar, "Super-mode OFDM with index modulation," arXiv e-prints, p. arXiv:2006.07765, Jun 2020.

[7] S. Darlington, "Pulse transmission," Patent US2 678 997A, Dec., 1949.

[8] X. Ouyang and J. Zhao, "Orthogonal chirp division multiplexing," IEEE Trans. Commun., vol. 64, no. 9, pp. 3946-3957, Sep. 2016.

[9] A. Şahin, N. Hosseini, H. Jamal, and D. W. Matolak, "DFT-spreadOFDM based chirp transmission," arXiv e-prints, p. arXiv:2008.03766, Aug. 2020.

[10] M. Golay, "Complementary series," IRE Trans. Inf. Theory, vol. 7, no. 2, pp. 82-87, Apr. 1961.

[11] J. A. Davis and J. Jedwab, "Peak-to-mean power control in OFDM, Golay complementary sequences, and Reed-Muller codes," IEEE Trans. Inf. Theory, vol. 45, no. 7, pp. 2397-2417, Nov. 1999.

[12] A. Şahin and R. Yang, "An uplink control channel design with complementary sequences for unlicensed bands," IEEE Trans. Wireless Commun., vol. 19, no. 10, pp. 6858-6870, Jul. 2020.

[13] J. Proakis and M. Salehi, Fundamentals of Communication Systems. Pearson Education, 2013.

[14] H. Sari, G. Karam, and I. Jeanclaude, "Transmission techniques for digital terrestrial TV broadcasting," IEEE Commun. Mag., vol. 33, no. 2, pp. 100-109, Feb. 1995.

[15] M. G. Parker, K. G. Paterson, and C. Tellambura, "Golay complementary sequences," in Wiley Encyclopedia of Telecommunications, 2003.

[16] K. G. Paterson and A. E. Jones, "Efficient decoding algorithms for generalized Reed-Muller codes," IEEE Trans. Commun., vol. 48, no. 8, pp. 1272-1285, Aug. 2000.

[17] K. Schmidt and A. Finger, "Simple maximum-likelihood decoding of generalized first-order Reed-Muller codes," IEEE Commun. Lett., vol. 9, no. 10 , pp. 912-914, Oct. 2005. 